\title{
Eigenvalue Asymptotics for the Maass Hamiltonian with Decreasing Electric Potentials
}

\author{
Shin-ichi SHIRAI*
}

\begin{abstract}
We study the eigenvalue distribution in the spectral gaps of the Maass Hamiltonian with electric potential $V$. For a real constant $B$, the (unperturbed) Maass Hamiltonian is given by

$$
H(0)=y^{2}\left(\frac{1}{\sqrt{-1}} \frac{\partial}{\partial x}-\frac{B}{y}\right)^{2}-y^{2} \frac{\partial^{2}}{\partial y^{2}},
$$

where $\mathbf{H}=\{(x, y) \mid x \in \mathbf{R}, y>0\}$ is the hyperbolic plane. The spectrum of the Maass Hamiltonian consists of the two disjoint parts: the continuous part and the discrete Landau levels (a finite number of eigenvalues of infinite multiplicity) if $|B|>1 / 2$. Following the argument as in Raikov, G. D. and Warzel, S. ["Quasi-classical versus non-classical spectral asymptotics for magnetic Schrödinger operators with decreasing potentials", Rev. Math. Phys., vol. 14, no. 10, (2002), 1051-1072], we obtain the asymptotic distribution of the number of discrete spectrum of $H(V)=H(0)+V$ near each discrete Landau level when $V$ is real-valued, asymptotically spherically symmetric and satisfies some decay estimates near infinity, or $V$ is compactly supported.
\end{abstract}

\section{$\S 1 . \quad$ Introduction and Results}

We consider the Maass Hamiltonian

$$
H(0)=y^{2}\left(\frac{1}{\sqrt{-1}} \frac{\partial}{\partial x}-\frac{B}{y}\right)^{2}-y^{2} \frac{\partial^{2}}{\partial y^{2}}
$$

acting in $L^{2}(\mathbf{H})$, where $B$ is a real constant and $\mathbf{H}=\{z=(x, y) \mid x \in \mathbf{R}, y>0\}$ is the hyperbolic plane. The Riemannian measure on $\mathbf{H}$ is given by $d x d y / y^{2}$

Communicated by T. Kawai. Received February 19, 2003. Revised July 7, 2003, October 31, 2003, December 22, 2003, February 27, 2004.

2000 Mathematics Subject Classification(s): Primary 35P20; Secondary 58J50.

*Department of Mathematics, Ritsumeikan University, Kusatsu, Shiga 525-77, Japan. 
and the hyperbolic distance $d\left(z, z_{0}\right)$ on $\mathbf{H}$ is given by $\cosh \left(d\left(z, z_{0}\right)\right)=(\mid x-$ $\left.\left.x_{0}\right|^{2}+y^{2}+y_{0}^{2}\right) /\left(2 y y_{0}\right)$ for any $z=(x, y), z_{0}=\left(x_{0}, y_{0}\right) \in \mathbf{H}$.

The operator $H(0)$ has a physical interpretation as the Hamiltonian which governs a non-relativistic, charged particle moving on $\mathbf{H}$ under the influence of the magnetic field of constant strength $B$ perpendicular to $\mathbf{H}$.

The spectral properties of the Maass Hamiltonian has been investigated by many authors (See, [Roe], [Els], [Fay], [Gro], [C-H], [Com], [A-P], and references therein). We recall some basic results. The Maass Hamiltonian $H(0)$ is essentially self-adjoint on $C_{0}^{\infty}(\mathbf{H})$, the set of all complex-valued, smooth functions with compact support on $\mathbf{H}$ ([Roe], Satz 3.2). (In what follows we use the same notation for an operator and its operator closure if there is no fear of confusion.) The spectrum of $H(0)$ consists of the absolutely continuous part $\left[B^{2}+1 / 4, \infty\right)$ and the discrete Landau levels $\left\{E_{n}\right\}_{n=0}^{N(|B|-1 / 2)}$, where $E_{n}=(2 n+1)|B|-n(n+1)$ and $N(x)$ denotes the largest integer less than $x$. In case $|B| \leq 1 / 2$, the set of discrete Landau levels is empty. If $|B|>1 / 2$, each of $E_{n} \mathrm{~s}$ is an eigenvalue of infinite multiplicity. In what follows we may restrict ourselves to the case $B>1 / 2$, provided we are concerned with the discrete Landau levels, since the Maass Hamiltonian $H(0)$ with $B$ is unitarily equivalent to the one with $-B$ via the transform $(x, y) \mapsto(-x, y)$.

For a measurable function $V$ on $\mathbf{H}$, we say $V$ decays at infinity if for any $\varepsilon>0$ there exists a compact subset $K$ of $\mathbf{H}$ such that $|V(x, y)|<\varepsilon$ outside $K$. Any bounded, measurable function $V$ decaying at infinity is relatively compact with respect to $H(0)$ (See [I-S], Lemma 3.10), so the operator

$$
H(V)=H(0)+V
$$

is a well-defined self-adjoint operator when $V$ is real-valued, and the essential spectrum of $H(V)$ coincides with that of $H(0)$ (See [R-S], Vol. IV). (Note that, examining the proof, one can easily find that Lemma 3.10 in [I-S] is still valid if we drop the continuity condition of $V$.) Then the perturbed operator $H(V)$ may have the discrete spectrum (i.e., discrete eigenvalues of finite multiplicity) in the spectral gaps.

The purpose of this paper is to obtain the asymptotic distribution of the number of the discrete spectrum near $E_{n}$ s. To formulate our results, we make the following condition $(\mathrm{V})_{\varepsilon}$ on the purterbation $V$.

$(\mathrm{V})_{\varepsilon}$ The perturbation $V$ is a real-valued, bounded, measurable and non-negative function on $\mathbf{H}$. Moreover, there exist $z_{0} \in \mathbf{H}$ and positive constants $\varepsilon$ and $C_{V}$ such that the asymptotic relation

$$
\lim _{d\left(z, z_{0}\right) \rightarrow \infty} \exp \left(\varepsilon d\left(z, z_{0}\right)\right) V(z)=C_{V}
$$


holds, where $d$ is the hyperbolic distance introduced at the beginning of this section.

Let $n$ be any non-negative integer $n$ satisfying $0 \leq n \leq N(B-1 / 2)$ and let $\varepsilon>0$. We introduce the notations

$$
\beta_{n}=2 B-2 n-1(>0)
$$

and

$$
\Theta_{n}(\varepsilon)=\frac{\Gamma\left(\beta_{n}+\varepsilon\right) \Gamma\left(\beta_{n}+n+1\right)}{\Gamma\left(\beta_{n}\right) \Gamma(n+1) \Gamma\left(\beta_{n}+1\right)} F_{2}\left(\beta_{n}+\varepsilon ;-n,-n ; \beta_{n}+1, \beta_{n}+1 ; 1,1\right) .
$$

Here, $\Gamma(z)=\int_{0}^{\infty} e^{-t} t^{z-1} d t$ is the gamma function and

$$
F_{2}\left(a ; b, b^{\prime} ; c, c^{\prime} ; x, y\right)=\sum_{l, m=0}^{\infty} \frac{(a)_{l+m}(b)_{l}\left(b^{\prime}\right)_{m}}{(c)_{l}\left(c^{\prime}\right)_{m}} \frac{x^{l} y^{m}}{l ! m !}
$$

is the Appell hypergeometric series (See [G-R], Section 9.18 and see also [Sla], Section 8) and $(x)_{0}=1$ and $(x)_{m}=x(x+1) \cdots(x+m-1)$ if $m \geq 1$. We note that, because of the parameter $-n$, the Appell series in the expressions of $\Theta_{n}(\varepsilon)$ terminates and it turns out that $\Theta_{n}(\varepsilon)$ is positive (See Lemma 2.2 below).

For any real numbers $a, b$ and for any self-adjoint operator $T$ acting in a Hilbert space, we set

$$
N(a<T<b)=\operatorname{dim} \operatorname{ran}\left(P_{T}((a, b))\right),
$$

where $P_{T}(I)$ stands for the the spectral projection for $T$ on an open interval $I$.

The main results of this paper are the following two theorems.

Theorem 1.1. Assume that $|B|>1 / 2$. Let $\left\{E_{n}\right\}_{n=0}^{N(|B|-1 / 2)}$ be as above. Let $E^{\prime}$ be any point between $E_{n}$ and $E_{n+1}$, where we set $E_{n+1}=B^{2}+1 / 4$ for $n=N(|B|-1 / 2)$. Then the condition $(V)_{\varepsilon}$ implies that

$$
\begin{aligned}
& N\left(E_{n}+E<H(V)<E^{\prime}\right) \\
& \quad=\frac{1}{4 \pi}\left(\Theta_{n}(\varepsilon)\right)^{1 / \varepsilon} \operatorname{Vol}_{\mathbf{H}}\{z \in \mathbf{H} \mid V(z)>E\}(1+o(1))
\end{aligned}
$$

as $E \searrow 0$, where $\mathrm{Vol}_{\mathbf{H}}$ is the Riemannian volume on $\mathbf{H}$.

For any $z_{0} \in \mathbf{H}$, we denote by $F_{T, t, z_{0}}$ the characteristic function on the set $\left\{z \in \mathbf{H} \mid t \leq d\left(z_{0}, z\right) \leq T\right\}$. 
Theorem 1.2. Assume that $|B|>1 / 2$ and $V$ is bounded, measurable, non-negative on $\mathbf{H}$ and decays at infinity. Let $E^{\prime}$ be any point between $E_{n}$ and $E_{n+1}$, where we set $E_{n+1}=B^{2}+1 / 4$ for $n=N(|B|-1 / 2)$. Let $z_{0} \in \mathbf{H}$ and $0 \leq t<T$. Then the following assertions hold:

(i) If there exists a positive constant $c$ such that $0 \leq V(z) \leq c F_{T, t, z_{0}}(z)$ holds for all $z \in \mathbf{H}$, then we have

$$
\left|\log \tanh ^{2}(T / 2)\right| \limsup _{E \searrow 0} N\left(E_{n}+E<H(V)<E^{\prime}\right) /|\log E| \leq 1 .
$$

(ii) If there exists a positive constant $c$ such that $c F_{T, t, z_{0}}(z) \leq V(z)$ holds for all $z \in \mathbf{H}$. then we have

$$
\left|\log \tanh ^{2}(T / 2)\right| \liminf _{E \backslash 0} N\left(E_{n}+E<H(V)<E^{\prime}\right) /|\log E| \geq 1 .
$$

(iii) In particular, if there exist positive constants $c, c^{\prime}$ such that $c F_{T, t, z_{0}}(z)$ $\leq V(z) \leq c^{\prime} F_{T, t, z_{0}}(z)$ holds for all $z \in \mathbf{H}$, then we have

$$
\left|\log \tanh ^{2}(T / 2)\right| \lim _{E \searrow 0} N\left(E_{n}+E<H(V)<E^{\prime}\right) /|\log E|=1 .
$$

We canonically identify any point $z=(x, y) \in \mathbf{H}$ with $z=x+\sqrt{-1} y$ in the complex upper-half plane.

Let $\mathrm{SL}(2, \mathbf{R})$ be the special linear group of $2 \times 2$ real matrices, which acts on $\mathbf{H}$ transitively and isometrically as the linear fractional transform $z \mapsto \gamma z=$ $(a z+b) /(c z+d)$ if $\gamma=\left(\begin{array}{l}a b \\ c d\end{array}\right) \in \mathrm{SL}(2, \mathbf{R})$. Fix $z_{0}=x_{0}+y_{0} \sqrt{-1} \in \mathbf{H}$ and set $\lambda=\sqrt{y_{0}}, a=x_{0} / y_{0}$ and $\gamma=\left(\begin{array}{cc}\lambda & \lambda a \\ 0 & \lambda^{-1}\end{array}\right)$. One can observe that $\gamma \in \operatorname{SL}(2, \mathbf{R})$ and $\gamma \sqrt{-1}=\lambda^{2} a+\lambda^{2} \sqrt{-1}=x_{0}+y_{0} \sqrt{-1}=z_{0}$. If we define a unitary operator $S$ acting on $L^{2}(\mathbf{H})$ by $(S f)(z)=f(\gamma z)=f\left(\lambda^{2}(x+a), \lambda^{2} y\right)$, we can find that $\left(S^{-1} f\right)(z)=f\left(x / \lambda^{2}-a, y / \lambda^{2}\right), S^{-1} \frac{\partial}{\partial x} S=\lambda^{2} \frac{\partial}{\partial x}, S^{-1} \frac{\partial}{\partial y} S=\lambda^{2} \frac{\partial}{\partial y}$ hold and the multiplication operator $g$ transforms as $S^{-1} g S=\left(S^{-1} g\right)=g\left(\gamma^{-1}\right.$. $)$ on $C_{0}^{\infty}(\mathbf{H})$, from which we can deduce that the operator $H(0)$ commutes with $S$. Then we have the unitary equivalence $S^{-1} H(V) S=H(0)+V\left(\gamma^{-1} \cdot\right)=H\left(V\left(\gamma^{-1} \cdot\right)\right)$. Hence it is enough to prove Theorem 1.1 and Theorem 1.2 in the case of $z_{0}=$ $\sqrt{-1}$.

Let $\mathbf{D}$ be the Poincaré disk $\left\{w=r e^{i \theta} \mid 0 \leq r<1,0 \leq \theta<2 \pi\right\}$ equipped with the standard measure $4 r\left(1-r^{2}\right)^{-2} d r d \theta$. The Cayley transform $A$ is defined by $A z=(z-\sqrt{-1}) /(z+\sqrt{-1})$ for each $z \in \mathbf{H}$, and $A$ defines an isometric diffeomorphism between $\mathbf{H}$ and $\mathbf{D}$, so it induces the unitary transform $A_{*}$ from $L^{2}(\mathbf{H})$ to $L^{2}(\mathbf{D})$ by $f(z) \mapsto f\left(A^{-1} w\right)$. For any $w=r e^{\sqrt{-1} \theta} \in \mathbf{D}$, the 
distance $d_{\mathbf{D}}(w, 0)$ on $\mathbf{D}$ is given by $\log [(1+r) /(1-r)]$, which coincides with $d\left(A^{-1} w, \sqrt{-1}\right)$ on $\mathbf{H}$. In the sequel, we shall identify $\mathbf{H}$ and $\mathbf{D}$ via $A$.

We note that, in the case of $z_{0}=\sqrt{-1}$, the asymptotic relation (1.1) is equivalent to the condition that

$$
\lim _{r \nearrow 1} \frac{V\left(A^{-1} w\right)}{\left(1-r^{2}\right)^{\varepsilon}}=4^{-\varepsilon} C_{V}
$$

holds uniformly in $\theta$ for $w=r e^{\sqrt{-1} \theta} \in \mathbf{D}$, which follows from the relation $1-r^{2}=\cosh ^{-2}\left(d_{\mathbf{D}}(w, 0) / 2\right)$.

Remark 1.3. Let $V$ satisfy $(\mathrm{V})_{\varepsilon}$ for some $\varepsilon>0$ and let $F_{T, t, z_{0}}$ be the function as in Theorem 1.2. Then a simple calculation shows that

$$
\begin{aligned}
& \lim _{E \searrow 0} E^{1 / \varepsilon} \operatorname{Vol}_{\mathbf{H}}\{z \in \mathbf{H} \mid V(z)>E\}=\pi C_{V}^{1 / \varepsilon}, \\
& \lim _{E \searrow 0} \operatorname{Vol}_{\mathbf{H}}\left\{z \in \mathbf{H} \mid F_{T, t, z_{0}}(z)>E\right\}=4 \pi\left(\cosh ^{2} T-\cosh ^{2} t\right) .
\end{aligned}
$$

Remark 1.4. Our results are concerned with the asymptotic distribution of the discrete spectrum accumulating to each discrete Landau level $E_{n}$ from the right. Analogous results hold if we consider the eigenvalues of $H(-V)$ accumulating to $E_{n}$ from the left.

Unfortunately, the author has not obtained the result at the lower edge of the continuous spectrum of $H(0)$.

In the Euclidean case, Raikov ([Rai], [Rai2]) has obtained the asymptotic distribution of the number of the discrete spectrum near the boundary of the essential spectrum of the Schrödinger operators with constant magnetic fields and power-like decreasing electric potentials. In the two dimensional case, the leading asymptotics are independent of the level-number $n$, and behave quasiclassically, i.e., behave like $(B / 2 \pi) \operatorname{Vol}_{\mathbf{R}^{2}}\left\{x \in \mathbf{R}^{2} \mid V(x)>E\right\}$ as $E \searrow 0$ (See, e.g., [R-W], Remark 2.5). Here, $B$ is the strength of the constant magnetic field and $B /(2 \pi)$ is the density of states for the $n$-th Landau level of the Landau Hamiltonian.

Recently, several authors ([R-W], $[\mathrm{M}-\mathrm{R}])$ investigate the asymptotics for the case where the decay of the electric potentials $V$ is Gaussian or faster. They show that the asymptotics are non-classical if the decay of $V$ is faster than Gaussian (in an appropriate sense), or support of $V$ is compact. The leading asymptotics are independent of $n$, and in the case of compact support, they do not depend on $V$.

On the other hand, our result shows that the asymptotic behaviour of $N\left(E_{n}+E<H(V)<E^{\prime}\right)$ has the form (1.2) as $E \searrow 0$. The density of states 
of the Maass Hamiltonian can be found in [Com], Eq.(5.14)-(5.16), Eq.(B.19). In particular, the density of states for the $n$-th discrete Landau level is given by $\beta_{n} /(4 \pi)$, which depends on $n$. The quantity $\beta_{n} /(4 \pi)$ does not coincide with the leading coefficient $\Theta_{n}(\varepsilon)^{1 / \varepsilon} /(4 \pi)$ in (1.2) unless $\varepsilon=1$. For example, we find that

$$
\begin{aligned}
& \Theta_{0}(\varepsilon)=\beta_{0} \frac{\Gamma\left(\beta_{0}+\varepsilon\right)}{\Gamma\left(\beta_{0}+1\right)}, \\
& \Theta_{1}(\varepsilon)=\beta_{1} \frac{\Gamma\left(\beta_{1}+\varepsilon\right)}{\Gamma\left(\beta_{1}+1\right)}\left(1+\frac{\varepsilon(\varepsilon-1)}{\beta_{1}+1}\right), \\
& \Theta_{2}(\varepsilon)=\beta_{2} \frac{\Gamma\left(\beta_{2}+\varepsilon\right)}{\Gamma\left(\beta_{2}+1\right)}\left(1+\frac{\varepsilon(\varepsilon-1)}{\beta_{2}+1}+\frac{\varepsilon(\varepsilon-1)}{\beta_{2}+2}+\frac{\varepsilon^{2}(\varepsilon-1)^{2}}{2\left(\beta_{2}+1\right)\left(\beta_{2}+2\right)}\right),
\end{aligned}
$$

etc. Obviously, $\Theta_{n}(\varepsilon)^{1 / \varepsilon}$ depends on both $n$ and $\varepsilon$. So, this is different from the flat case.

Remark 1.5. By using some hypergeometric identities (See, e.g., [A-A-R], [Sla]), we can also express $\Theta_{n}(\varepsilon)$ as

$$
\frac{\Gamma\left(\beta_{n}+\varepsilon\right)}{\Gamma\left(\beta_{n}\right)}{ }_{3} F_{2}\left(\begin{array}{c}
-n, 1-\varepsilon, \varepsilon \\
\beta_{n}+1,1
\end{array} ; 1\right),
$$

where ${ }_{3} F_{2}$ is the (generalized) Gauss hypergeometric function (See Section 2 below). However, this expression is not used in this paper.

The organization of this paper is as follows: In Section 2, we recall some elementary results for the gamma function and the hypergeometric functions. In Section 3, we derive an integral representation of $\Theta_{n}(\varepsilon)$, from which the positivity of $\Theta_{n}(\varepsilon)$ obeys. In Section 4, following the line of argument as in [R-W], we reduce the problem for $H(V)$ to that for the associated compact operator $P_{n} V P_{n}$. Here, $P_{n}$ stands for the spectral projection of $H(0)$ corresponding to $E_{n}$. In Section 5 and Section 6 , we obtain the asymptotic distribution of the eigenvalues of $P_{n} V P_{n}$ when $V$ are the functions as in Theorems 1.1 and 1.2, respectively. In Section 7 and Section 8, we give proofs for Theorem 1.1 and Theorem 1.2, respectively.

\section{$\S 2$. Preliminaries}

For later use, we prepare some elementary formulae for special functions. However, all results in this section are well-known in special function theory (See, e.g., [A-A-R], [Sla], [Leb] and [G-R]). We also show the positivity of the coefficient $\Theta_{n}(\varepsilon)$. 
The hypergeometric function ${ }_{p} F_{q}$ is given by

$$
{ }_{p} F_{q}\left(\begin{array}{l}
x_{1} x_{2} \ldots x_{p} \\
y_{1} y_{2} \ldots y_{q}
\end{array} ; z\right)=\sum_{m=0}^{\infty} \frac{\left(x_{1}\right)_{m}\left(x_{2}\right)_{m} \ldots\left(x_{p}\right)_{m}}{\left(y_{1}\right)_{m}\left(y_{2}\right)_{m} \ldots\left(y_{q}\right)_{m}} \frac{z^{m}}{m !} .
$$

Lemma 2.1. Let $\Gamma(z)$ be the gamma function and let $(a)_{m}$ as in Section 1. Then we have the following assertions:

(i) For any real numbers $\alpha, \beta$, we have

$$
\lim _{k \rightarrow \infty} k^{\beta-\alpha} \frac{\Gamma(k+\alpha)}{\Gamma(k+\beta)}=1 .
$$

(ii) If $x$ is not a non-positive integer, we have $(x)_{m}=\Gamma(x+m) / \Gamma(x)$ and $(-x)_{m}=(-1)^{m} \Gamma(x+1) / \Gamma(x-m+1)$. For any non-negative integer $n$, we have

$$
(-n)_{m}=\left\{\begin{array}{cc}
(-1)^{m} \Gamma(n+1) / \Gamma(n-m+1) & \text { if } 0 \leq m \leq n, \\
0 & \text { if } m \geq n+1 .
\end{array}\right.
$$

(iii) Let $\Re \gamma>\Re \beta>0$ and $|\arg (1-z)|<\pi$. Then we have

$$
{ }_{2} F_{1}\left(\begin{array}{c}
\alpha, \beta \\
\gamma
\end{array} ; z\right)=(1-z)^{-\alpha}{ }_{2} F_{1}\left(\begin{array}{c}
\alpha, \gamma-\beta \\
\gamma
\end{array} ; \frac{z}{z-1}\right) .
$$

Here, $\Re$ and arg stand for the real part and the argument of a complex number, respectively.

Proof. The assertion (i) follows from the Stirling asymptotic formula (e.g., [Leb], Section 1.2, Eq. 1.2.2 and Section 1.4, Eq. 1.4.23). The assertion (ii) is obvious by definition and the assertion (iii) is well-known (See, e.g., [Leb], Section 9.5, Eq. 9.5.1).

In the rest of this section we show the positivity of the asymptotic coefficient $\Theta_{n}(\varepsilon)$ as we stated in Section 1.

The Laguerre polynomial is given by

$$
\begin{aligned}
L_{n}^{\alpha}(x) & =\frac{1}{n !} e^{x} x^{-\alpha}\left(\frac{d}{d x}\right)^{n}\left(e^{-x} x^{n+\alpha}\right) \\
& =\sum_{m=0}^{n}(-1)^{m}\left(\begin{array}{c}
n+\alpha \\
n-m
\end{array}\right) \frac{x^{m}}{m !} \\
& =\left(\begin{array}{c}
n+\alpha \\
n
\end{array}\right){ }_{1} F_{1}\left(\begin{array}{c}
-n \\
\alpha+1
\end{array} ; x\right)
\end{aligned}
$$

(See [G-R], Section 8.97). 
Lemma 2.2. Let $n$ be a non-negative integer and let $\varepsilon>0$. Then we have

$$
\begin{aligned}
\Theta_{n}(\varepsilon) & =\frac{\Gamma\left(\beta_{n}+\varepsilon\right) \Gamma\left(\beta_{n}+n+1\right)}{\Gamma\left(\beta_{n}\right) \Gamma(n+1) \Gamma\left(\beta_{n}+1\right)} F_{2}\left(\beta_{n}+\varepsilon ;-n,-n ; \beta_{n}+1, \beta_{n}+1 ; 1,1\right) \\
& =\frac{\beta_{n} \Gamma(n+1)}{\Gamma\left(\beta_{n}+n+1\right)} \int_{0}^{\infty} t^{\beta_{n}+\varepsilon-1} e^{-t} L_{n}^{\beta_{n}}(t)^{2} d t
\end{aligned}
$$

In particular, the integral ensures the positivity of $\Theta_{n}(\varepsilon)$.

Proof. We show the equality (2.3) in the same way as in the proof of Lemma 1 in $[\mathrm{S}-\mathrm{H}]$. We note that the Appell series in (2.3) converges because of the parameter $-n$. It follows from (2.2) that

$$
\begin{aligned}
& \int_{0}^{\infty} t^{\beta_{n}+\varepsilon-1} e^{-t} L_{n}^{\beta_{n}}(t)^{2} d t \\
= & \left(\begin{array}{c}
n+\beta_{n} \\
n
\end{array}\right)^{2} \int_{0}^{\infty} t^{\beta_{n}+\varepsilon-1} e^{-t}{ }_{1} F_{1}\left(\begin{array}{c}
-n \\
\beta_{n}+1
\end{array} ; t\right)^{2} d t \\
= & \left(\begin{array}{c}
n+\beta_{n} \\
n
\end{array}\right)^{2} \sum_{l, m=0}^{n} \frac{(-n)_{l}(-n)_{m}}{\left(\beta_{n}+1\right)_{l}\left(\beta_{n}+1\right)_{m}} \frac{1}{l ! m !} \int_{0}^{\infty} t^{\beta_{n}+\varepsilon+l+m-1} e^{-t} d t \\
= & \left(\begin{array}{c}
n+\beta_{n} \\
n
\end{array}\right)^{2} \sum_{l, m=0}^{n} \frac{(-n)_{l}(-n)_{m}}{\left(\beta_{n}+1\right)_{l}\left(\beta_{n}+1\right)_{m}} \frac{1}{l ! m !} \Gamma\left(\beta_{n}+\varepsilon+l+m\right) \\
= & \left(\begin{array}{c}
n+\beta_{n} \\
n
\end{array}\right)^{2} \Gamma\left(\beta_{n}+\varepsilon\right) \sum_{l, m=0}^{n} \frac{(-n)_{l}(-n)_{m}\left(\beta_{n}+\varepsilon\right)_{l+m}}{\left(\beta_{n}+1\right)_{l}\left(\beta_{n}+1\right)_{m}} \frac{1}{l ! m !} \\
= & \left(\begin{array}{c}
n+\beta_{n} \\
n
\end{array}\right)^{2} \Gamma\left(\beta_{n}+\varepsilon\right) F_{2}\left(\beta_{n}+\varepsilon ;-n,-n ; \beta_{n}+1, \beta_{n}+1 ; 1,1\right),
\end{aligned}
$$

where we used Lemma 2.1 in the fourth equality. Then the result follows since $\Gamma\left(\beta_{n}+1\right)=\beta_{n} \Gamma\left(\beta_{n}\right)$ and

$$
\left(\begin{array}{c}
n+\beta_{n} \\
n
\end{array}\right)^{2} \Gamma\left(\beta_{n}+\varepsilon\right)=\frac{\Gamma\left(\beta_{n}+\varepsilon\right) \Gamma\left(\beta_{n}+n+1\right)^{2}}{\Gamma(n+1)^{2} \Gamma\left(\beta_{n}+1\right)^{2}} .
$$




\section{$\S 3 . \quad$ Reduction to a Single Landau-level Eigenspace}

In this section, following the argument as in Section 3 in $[\mathrm{R}-\mathrm{W}]$, we reduce the eigenvalue asymptotics for $H(V)$ near $E_{n}$ to that for the compact operator $P_{n} V P_{n}$ near 0 . Here, $P_{n}$ stands for the spectral projection of $H(0)$ corresponding to $E_{n}$.

For any real numbers $a, b$ and for any self-adjoint operator $T$, we denote

$$
\begin{aligned}
& N(a<T)=\operatorname{dim} \operatorname{ran}\left(P_{T}((a, \infty))\right), \\
& N(T<b)=\operatorname{dim} \operatorname{ran}\left(P_{T}((-\infty, b))\right) .
\end{aligned}
$$

Here, $P_{T}$ stands for the spectral projection for $T$.

Lemma 3.1. Let $T_{1}$ and $T_{2}$ be compact operators acting on a Hilbert space. Then for any $s>0$ and for any $\delta>0$ with $0<\delta<1$, we have

$$
\begin{aligned}
& N\left( \pm T_{1}>s(1+\delta)\right)-N\left(\mp T_{2}>s \delta\right) \\
& \quad \leq N\left( \pm\left(T_{1}+T_{2}\right)>s\right) \\
& \quad \leq N\left( \pm T_{1}>s(1-\delta)\right)+N\left( \pm T_{2}>s \delta\right),
\end{aligned}
$$

respectively.

Proof. This is a basic result in spectral theory for compact operators. See, e.g., [B-S], Chap. 11.

Lemma 3.2. $\quad$ Let $T$ be a self-adjoint operator acting in a Hilbert space and assume that the resolvent set of $T$ contains an interval $[\alpha, \beta]$. Assume that $V$ is non-negative, bounded and relatively compact with respect to $T$. Then we have

$$
\begin{aligned}
N(\alpha<T+V<\beta)= & N\left(V^{1 / 2}(\alpha-T)^{-1} V^{1 / 2}>1\right) \\
& -N\left(V^{1 / 2}(\beta-T)^{-1} V^{1 / 2}>1\right)-\operatorname{dim} \operatorname{ker}(T+V-\beta) .
\end{aligned}
$$

Proof. This is an easy consequence of the (generalized) Birman-Schwinger principle (e.g., [A-D-H], Theorem 1.3, [Bir], Proposition 1.5). However, we give a proof for the sake of completeness.

Let $E \in[\alpha, \beta]$. The Birman-Schwinger kernel is given by $X(E)=V^{1 / 2}(E-$ $T)^{-1} V^{1 / 2}$. Then the B-S principle says that an eigenvalue $E$ of $T+\lambda V(\lambda>0)$ of multiplicity $m$ corresponds to an eigenvalue $1 / \lambda$ of $X(E)$ of multiplicity $m$. Thus we have

$$
\begin{aligned}
\sum_{0<\lambda<1} \operatorname{dim} \operatorname{ker}(T+\lambda V-E) & =\sum_{0<\lambda<1} \operatorname{dim} \operatorname{ker}(X(E)-1 / \lambda) \\
& =N(X(E)>1) .
\end{aligned}
$$


On the other hand, we can deduce that each eigenvalue of $X(E)$ is monotonically decreasing in $E$ since the non-negativity of $V$ implies that

$$
\frac{\partial}{\partial E} V^{1 / 2}(E-T)^{-1} V^{1 / 2}=-V^{1 / 2}(E-T)^{-2} V^{1 / 2} \leq 0 .
$$

Then it follows from the B-S principle and the analytic perturbation theory (e.g., [R-S], vol. IV) that each eigenvalue of $T+\lambda V$ is monotonically increasing in $\lambda$ (See $[\mathrm{A}-\mathrm{D}-\mathrm{H}]$, Theorem 1.5, and see also the argument after Proposition 1.5 in [Bir] $)$. Then we have

$$
\begin{aligned}
N(\alpha<T+V<\beta) & \\
= & \sum_{0<\lambda<1} \operatorname{dim} \operatorname{ker}(T+\lambda V-\alpha) \\
& -\sum_{0<\lambda<1} \operatorname{dim} \operatorname{ker}(T+\lambda V-\beta)-\operatorname{dim} \operatorname{ker}(T+V-\beta) .
\end{aligned}
$$

Then the result follows from (3.3) and (3.2) with $E=\alpha, \beta$.

Lemma 3.3. The operator $P_{n} V P_{n}$ is compact, and for any $\delta>0$ small enough, we have, as $E \searrow 0$,

$$
\begin{aligned}
& N\left((1-\delta) P_{n} V P_{n}>E\right)+O(1) \leq N\left(E_{n}+E<H(V)<E^{\prime}\right) \\
& \quad \leq N\left((1+\delta) P_{n} V P_{n}>E\right)+O(1) .
\end{aligned}
$$

Proof. The proof is similar to that of Proposition 4.2 in $[\mathrm{R}-\mathrm{W}]$. However, we give a proof for the sake of completeness.

The compactness of $P_{n} V P_{n}$ follows easily from the fact that $V(H(0)-z)^{-1}$ is compact ([I-S], Lemma 3.10). By Lemma 3.2, we have

$$
\begin{aligned}
N( & \left.E_{n}+E<H(V)<E^{\prime}\right) \\
= & N\left(V^{1 / 2}\left(E_{n}+E-H(0)\right)^{-1} V^{1 / 2}>1\right) \\
& -N\left(V^{1 / 2}\left(E^{\prime}-H(0)\right)^{-1} V^{1 / 2}>1\right) \\
& -\operatorname{dim} \operatorname{ker}\left(H(V)-E^{\prime}\right) \\
= & N\left(V^{1 / 2}\left(E_{n}+E-H(0)\right)^{-1} V^{1 / 2}>1\right)+O(1)
\end{aligned}
$$

as $E \searrow 0$. Let $Q_{n}=I-P_{n}$. We apply Lemma 3.1 with $T_{1}=V^{1 / 2}\left(E_{n}+E-\right.$ $H(0))^{-1} P_{n} V^{1 / 2}, T_{2}=V^{1 / 2}\left(E_{n}+E-H(0)\right)^{-1} Q_{n} V^{1 / 2}$ and $s=1$. Then (3.1) 
with upper sign yields

$$
\begin{aligned}
N( & \left.V^{1 / 2}\left(E_{n}+E-H(0)\right)^{-1} P_{n} V^{1 / 2}>1+\delta\right) \\
& -N\left(V^{1 / 2}\left(E_{n}+E-H(0)\right)^{-1} Q_{n} V^{1 / 2}<-\delta\right) \\
\leq & N\left(V^{1 / 2}\left(E_{n}+E-H(0)\right)^{-1} V^{1 / 2}>1\right) \\
\leq & N\left(V^{1 / 2}\left(E_{n}+E-H(0)\right)^{-1} P_{n} V^{1 / 2}>1-\delta\right) \\
& +N\left(V^{1 / 2}\left(E_{n}+E-H(0)\right)^{-1} Q_{n} V^{1 / 2}>\delta\right) .
\end{aligned}
$$

Since $H(0) \geq 1 / 2$ and the distance between the point $E_{n}$ and the rest of the spectrum of $H(0)$ is positive, we have, for small $E>0$,

$$
\inf \left\{\left|\left(E_{n}+E\right)-x\right| / x \mid x \in \sigma(H(0)) \backslash\left\{E_{n}\right\}\right\} \geq C_{n}>0
$$

for some constant $C_{n}$, where $\sigma(\cdot)$ stands for the spectrum. Hence, we have

$$
\begin{aligned}
& \left|E_{n}+E-H(0)\right|^{-1} Q_{n} \\
& \quad=\sum_{j \neq n}\left|E_{n}+E-E_{j}\right|^{-1} P_{j}+\int_{B^{2}+1 / 4}^{\infty}\left|E_{n}+E-\lambda\right|^{-1} d P_{H(0)}(\lambda) \\
& \quad \leq C_{n}\left(\sum_{j \neq n} E_{j}^{-1} P_{j}+\int_{B^{2}+1 / 4}^{\infty} \lambda^{-1} d P_{H(0)}(\lambda)\right) \\
& \quad \leq C_{n} H(0)^{-1} .
\end{aligned}
$$

Then, for each $\delta>0$ small enough, we have, as $E \searrow 0$,

$$
\begin{aligned}
N & \left( \pm V^{1 / 2}\left(E_{n}+E-H(0)\right)^{-1} Q_{n} V^{1 / 2}>\delta\right) \\
& \leq N\left(V^{1 / 2}\left|E_{n}+E-H(0)\right|^{-1} Q_{n} V^{1 / 2}>\delta\right) \\
& \leq N\left(V^{1 / 2} C_{n} H(0)^{-1} V^{1 / 2}>\delta\right) \\
& =O(1) .
\end{aligned}
$$

The result follows from (3.4)-(3.6).

We now introduce the angular-momentum eigenfunctions (i.e., eigenfunctions of the form $\left.e^{i k \theta} G_{k}(r)\right)$ for $H(V)$ and show that the eigenvalues of $P_{n} V P_{n}$ can be described in terms of these eigenfunctions.

Let $A$ be the Cayley transform. We define a unitary operator $U_{B}$ from $L^{2}(\mathbf{H})$ to $L^{2}(\mathbf{D})$ by

$$
\left(U_{B} f\right)(w)=\left(\frac{1-\bar{w}}{1-w}\right)^{B} f\left(A^{-1} w\right)
$$


for any $f \in L^{2}(\mathbf{H})$, where $1^{B}=1$. Then we have

$$
\begin{aligned}
& U_{B} H(0) U_{B}^{-1} \\
& \quad=-\frac{1}{4}\left(1-r^{2}\right)^{2}\left(\frac{\partial^{2}}{\partial r^{2}}+\frac{1}{r} \frac{\partial}{\partial r}+\frac{1}{r^{2}} \frac{\partial^{2}}{\partial \theta^{2}}\right)+i\left(1-r^{2}\right) \frac{\partial}{\partial \theta}-\left(1-r^{2}\right) B^{2}+B^{2}
\end{aligned}
$$

(See [Els], Satz 2.1 and see also [Fay], Theorem 1.1). Moreover, a complete set of orthogonal angular-momentum eigenfunctions $\left\{\varphi_{n k}\right\}_{k \geq-n}^{\infty}$ corresponding to the eigenvalue $E_{n}$ is known (See Satz 3.2 in [Els], Theorem 1.4 in [Fay], Eq.13 in [Gro2] and see also Eq.4.47 in [K-L]). Especially, the eigenfunction is given by

$$
\varphi_{n k}=\sqrt{C_{n k}} e^{i k \theta} r^{k}\left(1-r^{2}\right)^{B-n}{ }_{2} F_{1}\left(\begin{array}{c}
-n, k+\beta_{n}+n+1 \\
k+1
\end{array} ; r^{2}\right)
$$

in the case of $k \geq 0$, where

$$
C_{n k}=\frac{\beta_{n} \Gamma\left(k+\beta_{n}+n+1\right) \Gamma(k+n+1)}{4 \pi \Gamma(n+1) \Gamma(k+1)^{2} \Gamma\left(\beta_{n}+n+1\right)} .
$$

Note that, because of the parameter $-n$, the hypergeometric function above is a polynomial with respect to $r^{2}$, in fact, we can find that

$$
P_{n}^{\left(k, \beta_{n}\right)}\left(1-r^{2}\right)=\left(\begin{array}{c}
n+k \\
n
\end{array}\right){ }_{2} F_{1}\left(\begin{array}{c}
-n, k+\beta_{n}+n+1 \\
k+1
\end{array} ; r^{2}\right),
$$

where the Jacobi polynomial $P_{n}^{(\alpha, \beta)}$ is given by

$$
P_{n}^{(\alpha, \beta)}(x)=\frac{(-1)^{n}}{2^{n} n !}(1-x)^{-\alpha}(1+x)^{-\beta}\left(\frac{d}{d x}\right)^{n}\left((1-x)^{\alpha+n}(1+x)^{\beta+n}\right)
$$

and we set

$$
\left(\begin{array}{c}
n \\
m
\end{array}\right)=\frac{\Gamma(n+1)}{\Gamma(n-m+1) \Gamma(m+1)}
$$

(See [Leb], Section 4, p. 96, and for the relation between the Jacobi polynomial and the hypergeometric function, see also [G-R], Section 8.96, p. 1059).

In what follows we identify the operator $U_{B} H(0) U_{B}^{-1}$, the associated spectral projections, and the function $A_{*} V=V\left(A^{-1} \cdot\right)$ with $H(0), P_{n}$, and $V$, respectively. 
Lemma 3.4. Let $V$ be any bounded, measurable and spherically symmetric function on $\mathbf{D}$. The set of eigenvalues of the compact operator $P_{n} V P_{n}$ (acting on the range of $\left.P_{n}\right)$ is given by $\left\{\left(\varphi_{n k}, V \varphi_{n k}\right)\right\}_{k=-n}^{\infty}$, where $\varphi_{n k}$ is the eigenfunction of $H(0)$ as in $(3.7)$ and $(\cdot, \cdot)$ denotes the inner product on $L^{2}(\mathbf{D})$.

Proof. Because of the orthogonality with respect to the angular momentum and the symmetry of $V$, we have $\left(\varphi_{n k}, V \varphi_{n k^{\prime}}\right)=0$ if $k \neq k^{\prime}$. Then it follows that $P_{n} V P_{n} \varphi_{n k}=\left(\varphi_{n k}, V \varphi_{n k}\right) \varphi_{n k}$. The result follows from the completeness of $\left\{\varphi_{n k}\right\}_{k=-n}^{\infty}$ in the range of $P_{n}$.

\section{$\S 4$. Eigenvalue Asymptotics for $P_{n} V_{\varepsilon} P_{n}$}

In what follows we set $V_{\varepsilon}(w)=\left(1-|w|^{2}\right)^{\varepsilon}$ for any $\varepsilon>0$, and we set

$$
\gamma_{n k}(V)=\left(\varphi_{n k}, V \varphi_{n k}\right)
$$

for any function $V$ on $\mathbf{D}$ and for any $k \geq-n$. In the sequel, we investigate the asymptotic behaviour of the eigenvalues $\gamma_{n k}\left(V_{\varepsilon}\right)$ as $k \rightarrow \infty$, so we may assume that $k>0$ and $\varphi_{n k}$ is of the form (3.7).

Lemma 4.1. We have

$$
\begin{aligned}
& { }_{2} F_{1}\left(\begin{array}{cc}
-n k+\beta_{n}+n+1 ; r^{2} \\
k+1
\end{array}\right)^{2} \\
& =\sum_{l, m=0}^{n}(-1)^{l+m}\left(\begin{array}{c}
n \\
l
\end{array}\right)\left(\begin{array}{c}
n \\
m
\end{array}\right) \times \\
& \times \frac{\Gamma\left(\beta_{n}+n+1\right)^{2} \Gamma(k+1)^{2}}{\Gamma\left(\beta_{n}+n-l+1\right) \Gamma\left(\beta_{n}+n-m+1\right) \Gamma(k+1+l) \Gamma(k+1+m)} \times \\
& \times\left(1-r^{2}\right)^{2 n-l-m} r^{2(l+m)} .
\end{aligned}
$$

Proof. By Lemma 2.1 (ii) with $\beta=k+\beta_{n}+n+1, \gamma=k+1, \gamma-\beta=$ $-\left(\beta_{n}+n\right)$, and $z=r^{2}$, we have

$$
{ }_{2} F_{1}\left(\begin{array}{c}
-n \beta \\
\gamma
\end{array} r^{2}\right)=\left(1-r^{2}\right)^{n}{ }_{2} F_{1}\left(\begin{array}{c}
-n-\left(\beta_{n}+n\right) \\
k+1
\end{array} \frac{r^{2}}{r^{2}-1}\right) .
$$

Then the result follows from the series expression (2.1) and Lemma 2.1 (i). 
Lemma 4.2. Let $V=V(r)$ be bounded, continuous and spherically symmetric. Then we have

$$
\begin{aligned}
\gamma_{n k}(V)= & 4 C_{n k} \sum_{m, l=0}^{n}(-1)^{m+l}\left(\begin{array}{c}
n \\
m
\end{array}\right)\left(\begin{array}{c}
n \\
l
\end{array}\right) \times \\
& \times \frac{\Gamma\left(\beta_{n}+n+1\right)^{2} \Gamma(k+1)^{2}}{\Gamma\left(\beta_{n}+n-m+1\right) \Gamma\left(\beta_{n}+n-l+1\right) \Gamma(k+1+m) \Gamma(k+1+l)} \times \\
& \times \int_{0}^{1} t^{k+m+l}(1-t)^{\beta_{n}+2 n-m-l-1} V(\sqrt{t}) d t .
\end{aligned}
$$

In particular, with $V=V_{\varepsilon}$, we have

$$
\begin{aligned}
(4.2) \gamma_{n k}\left(V_{\varepsilon}\right) & =4 \pi C_{n k} \sum_{m, l=0}^{n}(-1)^{m+l}\left(\begin{array}{c}
n \\
m
\end{array}\right)\left(\begin{array}{l}
n \\
l
\end{array}\right) \\
& \times \frac{\Gamma\left(\beta_{n}+n+1\right)^{2} \Gamma(k+1)^{2}}{\Gamma\left(\beta_{n}+n-m+1\right) \Gamma\left(\beta_{n}+n-l+1\right) \Gamma(k+1+m) \Gamma(k+1+l)} \\
& \times \frac{\Gamma(k+m+l+1) \Gamma\left(\beta_{n}+2 n-m-l+\varepsilon\right)}{\Gamma\left(\beta_{n}+k+2 n+\varepsilon+1\right)} .
\end{aligned}
$$

Proof. $\quad$ By (3.7) and Lemma 4.1, we have

$$
\begin{aligned}
& \gamma_{n k}(V) \\
= & \int_{0}^{2 \pi} d \theta \int_{0}^{1} \frac{r d r}{\left(1-r^{2}\right)^{2}} V(r) C_{n k} r^{2 k}\left(1-r^{2}\right)^{2(B-n)} \times \\
& \times{ }_{2} F_{1}\left(\begin{array}{c}
-n k+\beta_{n}+n+1 \\
k+1
\end{array} r^{2}\right)^{2} \\
= & 8 \pi C_{n k} \sum_{m, l=0}^{n}(-1)^{m+l}\left(\begin{array}{c}
n \\
m
\end{array}\right)\left(\begin{array}{c}
n \\
l
\end{array}\right) \frac{\Gamma\left(\beta_{n}+n+1\right)^{2}}{\Gamma\left(\beta_{n}+n-m+1\right) \Gamma\left(\beta_{n}+n-l+1\right)} \times \\
& \times \frac{\Gamma(k+1)^{2}}{\Gamma(k+1+m) \Gamma(k+1+l)} \int_{0}^{1} r^{2(k+m+l)+1}\left(1-r^{2}\right)^{\beta_{n}+2 n-m-l-1} V(r) d r,
\end{aligned}
$$

where we used $\beta_{n}=2 B-2 n-1$ in the last equality. Then we have the first assertion by changing the variable $t=r^{2}$ in the last integral. 
The second assertion follows from

$$
\begin{aligned}
& \int_{0}^{1} t^{k+m+l}(1-t)^{\beta_{n}+2 n-m-l-1} V_{\varepsilon}(\sqrt{t}) d t \\
& \quad=\int_{0}^{1} t^{k+m+l}(1-t)^{\beta_{n}+2 n-m-l-1+\varepsilon} d t \\
& =B\left(k+m+l+1, \beta_{n}+2 n-m-l+\varepsilon\right) \\
& =\frac{\Gamma(k+m+l+1) \Gamma\left(\beta_{n}+2 n-m-l+\varepsilon\right)}{\Gamma\left(k+\beta_{n}+2 n+\varepsilon+1\right)},
\end{aligned}
$$

where $B(p, q)=\int_{0}^{1} t^{p-1}(1-t)^{q-1} d t$ is the beta function.

Lemma 4.3. For any $\varepsilon>0$, we have

$$
\begin{aligned}
& \lim _{k \rightarrow \infty} k^{\varepsilon} \gamma_{n k}\left(V_{\varepsilon}\right) \\
& \quad=\frac{\Gamma\left(\beta_{n}+\varepsilon\right)}{\Gamma\left(\beta_{n}\right)} \frac{\Gamma\left(\beta_{n}+n+1\right)}{\Gamma(n+1) \Gamma\left(\beta_{n}+1\right)} F_{2}\left(\beta_{n}+\varepsilon ;-n,-n ; \beta_{n}+1, \beta_{n}+1 ; 1,1\right) .
\end{aligned}
$$

Proof. By (4.2) and (3.8), we have

$$
\begin{aligned}
\gamma_{n k}\left(V_{\varepsilon}\right)= & \sum_{m, l=0}^{n}(-1)^{m+l}\left(\begin{array}{c}
n \\
m
\end{array}\right)\left(\begin{array}{c}
n \\
l
\end{array}\right) \frac{\beta_{n} \Gamma\left(\beta_{n}+n+1\right)}{\Gamma(n+1)} \times \\
& \times \frac{\Gamma\left(\beta_{n}+2 n-m-l+\varepsilon\right)}{\Gamma\left(\beta_{n}+n-m+1\right) \Gamma\left(\beta_{n}+n-l+1\right)} \times \\
& \times \frac{\Gamma\left(k+\beta_{n}+n+1\right) \Gamma(k+n+1) \Gamma(k+m+l+1)}{\Gamma(k+m+1) \Gamma(k+l+1) \Gamma\left(k+\beta_{n}+2 n+\varepsilon+1\right)} .
\end{aligned}
$$

Using Lemma 2.1 (iii), we have

$$
\lim _{k \rightarrow \infty} k^{\varepsilon} \frac{\Gamma\left(k+\beta_{n}+n+1\right) \Gamma(k+n+1) \Gamma(k+m+l+1)}{\Gamma(k+m+1) \Gamma(k+l+1) \Gamma\left(k+\beta_{n}+2 n+\varepsilon+1\right)}=1,
$$


since $\left(\beta_{n}+n+1\right)+(n+1)+(m+l+1)-(m+1)-(l+1)-\left(\beta_{n}+2 n+\varepsilon+1\right)=-\varepsilon$. Then it follows from (4.3) and (4.4) that

$$
\text { (4.5) } \begin{aligned}
& \lim _{k \rightarrow \infty} k^{\varepsilon} \gamma_{n k}\left(V_{\varepsilon}\right) \\
= & \frac{\beta_{n} \Gamma\left(\beta_{n}+n+1\right)}{\Gamma(n+1)} \sum_{l, m=0}^{n}(-1)^{l+m} \\
& \times\left(\begin{array}{c}
n \\
l
\end{array}\right)\left(\begin{array}{c}
n \\
m
\end{array}\right) \frac{\Gamma\left(\beta_{n}+2 n-l-m+\varepsilon\right)}{\Gamma\left(\beta_{n}+n-l+1\right) \Gamma\left(\beta_{n}+n-m+1\right)} \\
= & \frac{\beta_{n} \Gamma\left(\beta_{n}+n+1\right)}{\Gamma(n+1)} \sum_{i, j=0}^{n}(-1)^{i+j}\left(\begin{array}{c}
n \\
i
\end{array}\right)\left(\begin{array}{c}
n \\
j
\end{array}\right) \frac{\Gamma\left(\beta_{n}+i+j+\varepsilon\right)}{\Gamma\left(\beta_{n}+i+1\right) \Gamma\left(\beta_{n}+j+1\right)} \\
= & \frac{\beta_{n} \Gamma\left(\beta_{n}+n+1\right)}{\Gamma(n+1)} \frac{\Gamma\left(\beta_{n}+\varepsilon\right)}{\Gamma\left(\beta_{n}+1\right)^{2}} \sum_{i, j=0}^{n} \frac{(-1)^{i} \Gamma(n+1)}{\Gamma(n-i)^{j} \Gamma(n+1)} \\
= & \frac{\Gamma\left(\beta_{n}+i+j+\varepsilon\right)}{\Gamma\left(\beta_{n}+\varepsilon\right)} \frac{\Gamma\left(\beta_{n}+1\right)}{\Gamma\left(\beta_{n}+i+1\right)} \frac{\Gamma\left(\beta_{n}+1\right)}{\Gamma\left(\beta_{n}+j+1\right)} \frac{1}{i ! j !} \\
= & \frac{\beta_{n} \Gamma\left(\beta_{n}+n+1\right)}{\Gamma(n+1)} \frac{\Gamma\left(\beta_{n}+\varepsilon\right)}{\Gamma\left(\beta_{n}+1\right)^{2}} \sum_{i, j=0}^{n} \frac{(-n)_{i}(-n)_{j}\left(\beta_{n}+\varepsilon\right)_{i+j}}{\left(\beta_{n}+1\right)_{i}\left(\beta_{n}+1\right)_{j}} \frac{1}{i ! j !} \\
\Gamma(n+1) & \frac{\Gamma\left(\beta_{n}+\varepsilon\right)}{\Gamma\left(\beta_{n}+1\right)^{2}} F_{2}\left(\beta_{n}+\varepsilon ;-n,-n ; \beta_{n}+1, \beta_{n}+1 ; 1,1\right),
\end{aligned}
$$

where we set $i=n-l, j=n-m$ in the second equality and used Lemma 2.1 in the fourth equality. This proves the lemma.

\section{§5. Eigenvalue Asymptotics for the Potential Supported in an Annulus}

In this section we investigate the asymptotic behaviour of the eigenvalues $\gamma_{n k}\left(W_{r R}\right)$ as $k \rightarrow \infty$. Here, $W_{r R}$ stands for the characteristic function on the set $\left\{w=|w| e^{i \theta} \in \mathbf{D}|r \leq| w \mid \leq R\right\}$.

Lemma 5.1. Let $\beta$ be a real number and let $r, R$ satisfy the relation $0 \leq r<R<1$. If we define

$$
B_{r R}(K, \beta)=\int_{r}^{R} t^{K-1}(1-t)^{\beta-1} d t,
$$

the estimate

$$
C_{r, R, \beta} \frac{R^{K}}{K} \leq B_{r R}(K, \beta) \leq C_{r, R, \beta}^{\prime} \frac{R^{K}}{K}
$$


holds for any $K>0$ large enough. Here, the constants $C_{r, R, \beta}, C_{r, R, \beta}^{\prime}$ are independent of large $K$.

Proof. If $\beta>1$, we have

$$
\begin{aligned}
(1 & -R)^{\beta-1} \frac{R^{K}-r^{K}}{K}=(1-R)^{\beta-1} \int_{r}^{R} t^{K-1} d t \\
& \leq B_{r R}(K, \beta) \\
& \leq(1-r)^{\beta-1} \int_{r}^{R} t^{K-1} d t=(1-r)^{\beta-1} \frac{R^{K}-r^{K}}{K},
\end{aligned}
$$

since $(1-R)^{\beta-1} \leq(1-t)^{\beta-1} \leq(1-r)^{\beta-1}$ holds if $r \leq t \leq R$. Similarly, if $\beta \leq 1$, we have

$$
(1-r)^{\beta-1} \frac{R^{K}-r^{K}}{K} \leq B_{r R}(K, \beta) \leq(1-R)^{\beta-1} \frac{R^{K}-r^{K}}{K} .
$$

Thus we have

$$
\begin{aligned}
& \min \left\{(1-R)^{\beta-1},(1-r)^{\beta-1}\right\}\left(1-(r / R)^{K}\right) \frac{R^{K}}{K} \\
& \quad \leq B_{r R}(K, \beta) \\
& \quad \leq \max \left\{(1-R)^{\beta-1},(1-r)^{\beta-1}\right\}\left(1-(r / R)^{K}\right) \frac{R^{K}}{K},
\end{aligned}
$$

from which the lemma follows since $1 / 2<1-(r / R)^{K}<1$ holds for large $K$.

Lemma 5.2. Let $0 \leq r<R<1$ and let $W_{r R}$ be the characteristic function for the set $\left\{w=|w| e^{i \theta} \in \mathbf{D}|r \leq| w \mid \leq R\right\}$. Then we have

$$
\lim _{k \rightarrow \infty} \frac{\log \gamma_{n k}\left(W_{r R}\right)}{2 k \log R}=1
$$

Proof. By Lemma 4.2 with $V=W_{r R}$, we have

$$
\begin{aligned}
\gamma_{n k}\left(W_{r R}\right) & =\sum_{l, m=0}^{n} C_{m l}(k) \int_{0}^{1} t^{k+m+l}(1-t)^{\beta_{n}+2 n-m-l-1} W_{r R}(\sqrt{t}) d t \\
& =\sum_{l, m=0}^{n} C_{m l}(k) B_{r^{2}} R^{2}\left(k+m+l+1, \beta_{n}+2 n-m-l\right)
\end{aligned}
$$


where we set

$$
\begin{aligned}
& C_{m l}(k) \\
&= 4 \pi C_{n k}(-1)^{m+l}\left(\begin{array}{c}
n \\
m
\end{array}\right)\left(\begin{array}{c}
n \\
l
\end{array}\right) \frac{\Gamma\left(\beta_{n}+n+1\right)^{2}}{\Gamma\left(\beta_{n}+n-m+1\right)} \frac{\Gamma(k+1)^{2}}{\Gamma\left(\beta_{n}+n-l+1\right)} \times \\
& \times \frac{1}{\Gamma(k+1+m) \Gamma(k+1+l)} \\
&= \frac{(-1)^{m+l} \beta_{n}\left(\begin{array}{c}
n \\
m
\end{array}\right)\left(\begin{array}{c}
n \\
l
\end{array}\right) \Gamma\left(\beta_{n}+n+1\right)}{\Gamma(n+1) \Gamma\left(\beta_{n}+n-m+1\right) \Gamma\left(\beta_{n}+n-l+1\right)} \frac{\Gamma\left(k+\beta_{n}+n+1\right) \Gamma(k+n+1)}{\Gamma(k+1+m) \Gamma(k+l+1)} .
\end{aligned}
$$

In the rest of the proof, we denote by $\sum^{\prime}$ the summation over $l, m$ satisfying $0 \leq l \leq n, 0 \leq m \leq n$ and $l+m \geq 1$. It follows from (5.1) that

(5.2) $\log \gamma_{n k}\left(W_{r R}\right)$

$$
\begin{aligned}
= & \log \left[C_{00}(k) B_{r^{2} R^{2}}\left(k+1, \beta_{n}+2 n\right) \times\right. \\
& \left.\times\left(1+\sum, \frac{C_{m l}(k) B_{r^{2} R^{2}}\left(k+m+l+1, \beta_{n}+2 n-m-l\right)}{C_{00}(k) B_{r^{2} R^{2}}\left(k+1, \beta_{n}+2 n\right)}\right)\right] \\
= & \log C_{00}(k)+\log B_{r^{2} R^{2}}\left(k+1, \beta_{n}+2 n\right)+ \\
& +\log \left(1+\sum^{\prime} \frac{C_{m l}(k) B_{r^{2} R^{2}}\left(k+m+l+1, \beta_{n}+2 n-m-l\right)}{C_{00}(k) B_{r^{2} R^{2}}\left(k+1, \beta_{n}+2 n\right)}\right) .
\end{aligned}
$$

By Lemma 2.1 (iii), there exists $C_{n}>0$, independent of $k$, such that

$$
\begin{gathered}
\lim _{k \rightarrow \infty} k^{-\left(\beta_{n}+2 n\right)} C_{00}(k)=\frac{\beta_{n}}{\Gamma(n+1) \Gamma\left(\beta_{n}+n+1\right)}, \\
\left|C_{m l}(k)\right| \leq C_{n} k^{\beta_{n}+2 n-m-l}
\end{gathered}
$$

hold for large $k$. By Lemma 5.1 and (5.3), we have, for large $k>0$,

$$
\begin{aligned}
& \left|\sum^{\prime} \frac{C_{m l}(k) B_{r^{2} R^{2}}\left(k+m+l+1, \beta_{n}+2 n-m-l\right)}{C_{00}(k) B_{r^{2} R^{2}}\left(k+1, \beta_{n}+2 n\right)}\right| \\
& \quad \leq C_{r, R, \beta_{n}} \sum^{\prime} \frac{k^{\beta_{n}+2 n-m-l}}{k^{\beta_{n}+2 n}} \frac{R^{2(k+m+l+1)}}{k+m+l+1} \frac{k+1}{R^{2(k+1)}} \\
& \quad \leq C_{r, R, \beta_{n}}^{\prime} \sum^{\prime} k^{-m-l} \\
& \leq C_{r, R, \beta_{n}}^{\prime \prime} k^{-1}
\end{aligned}
$$

for some positive constants $C_{r, R, \beta_{n}}, C_{r, R, \beta_{n}}^{\prime}, C_{r, R, \beta_{n}}^{\prime \prime}$, independent of $k$, where we used the fact that the sum is finite $(l, m \leq n)$ in the last inequality. Then it 
follows from (5.2) and (5.4) that the rhs of (5.2) is equal to $2 k \log R+O(\log k)$ as $k \rightarrow \infty$, since (5.3) and Lemma 5.1 imply that

$$
\begin{aligned}
\log C_{00}(k) & =O(\log k), \\
\log B_{r^{2} R^{2}}\left(k+n, \beta_{n}+2 n\right) & =2 k \log R+O(\log k)
\end{aligned}
$$

as $k \rightarrow \infty$, respectively. This proves the lemma.

\section{$\S 6 . \quad$ Proof of Theorem 1.1}

Let $V_{\varepsilon}(w)=\left(1-|w|^{2}\right)^{\varepsilon}$ as in Section 4 and let $W_{r R}$ be the function as in the previous section. To the end of the paper, we identify any objects (e.g., function, point) on $\mathbf{H}$ with the corresponding ones on $\mathbf{D}$, via the Cayley transform $A$, stated just after Theorem 1.2 and in Section 3.

As we remarked just after Theorem 1.2, it is enough to show in the case of $z_{0}=\sqrt{-1}$, and the condition $(\mathrm{V})_{\varepsilon}$ implies that, for any $\delta>0$ small enough, there exists $R>0$ such that

$$
(1-\delta) C_{V}^{\prime} V_{\varepsilon}(w) \leq V(w) \leq(1+\delta) C_{V}^{\prime} V_{\varepsilon}(w)
$$

holds for any $w \in \mathbf{D}$ with $|w| \geq R$. Here, we set $C_{V}^{\prime}=4^{-\varepsilon} C_{V}$. Thus there exists $M>0$ such that

$$
(1-\delta) C_{V}^{\prime} V_{\varepsilon}(w)-M W_{0 R}(w) \leq V(w) \leq(1+\delta) C_{V}^{\prime} V_{\varepsilon}(w)+M W_{0 R}(w)
$$

holds for all $w \in \mathbf{D}$.

By Lemma 3.3, we have

$$
\begin{aligned}
& N\left(E_{n}+E<H(V)<E^{\prime}\right) \\
& \quad \geq N\left((1-\delta) P_{n} V P_{n}>E\right)+O(1) \\
& \quad \geq N\left((1-\delta) P_{n}\left((1-\delta) C_{V}^{\prime} V_{\varepsilon}-M W_{0 R}\right) P_{n}>E\right)+O(1),
\end{aligned}
$$

as $E \searrow 0$, where we used the lower half of (6.1) in the second inequality. Similarly, we have

$$
\begin{aligned}
& N\left(E_{n}+E<H(V)<E^{\prime}\right) \\
& \quad \leq N\left((1+\delta) P_{n}\left((1+\delta) C_{V}^{\prime} V_{\varepsilon}+M W_{0 R}\right) P_{n}>E\right)+O(1)
\end{aligned}
$$

as $E \searrow 0$. Because of the spherical symmetry of $V_{\varepsilon}$ and $W_{0 R}$, using Lemma 3.4 , we have

$$
\begin{aligned}
& N\left((1 \mp \delta) P_{n}\left((1 \mp \delta) C_{V}^{\prime} V_{\varepsilon} \mp M W_{0 R}\right) P_{n}>E\right) \\
& \quad=\sharp\left\{k \mid(1 \mp \delta)\left[\left((1 \mp \delta) C_{V}^{\prime} \gamma_{n k}\left(V_{\varepsilon}\right) \mp M \gamma_{n k}\left(W_{0 R}\right)\right)\right]>E\right\},
\end{aligned}
$$


where $\gamma_{k n}(\cdot)$ is as in (4.1) and $\sharp$ denotes the cardinality of the set. By Lemmas 4.3 and 5.2 , for any $\delta>0$, there exists $k_{\delta}>0$ such that

$$
\begin{aligned}
& (1-\delta)\left[\left((1-\delta) C_{V}^{\prime} \gamma_{n k}\left(V_{\varepsilon}\right)-M \gamma_{n k}\left(W_{0 R}\right)\right)\right] \\
& \quad \geq(1-2 \delta)(1-\delta) C_{V}^{\prime} \gamma_{n k}\left(V_{\varepsilon}\right) \\
& \quad \geq(1-3 \delta)(1-\delta) C_{V}^{\prime} \Theta_{n}(\varepsilon) k^{-\varepsilon}
\end{aligned}
$$

for all $k \geq k_{\delta}$ (we take $\delta$ small enough). Similarly, we have, for any $\delta>0$,

$$
\begin{aligned}
(1 & +\delta)\left[\left((1+\delta) C_{V}^{\prime} \gamma_{n k}\left(V_{\varepsilon}\right)+M \gamma_{n k}\left(W_{0 R}\right)\right)\right] \\
& \leq(1+2 \delta)(1+\delta) C_{V}^{\prime} \gamma_{n k}\left(V_{\varepsilon}\right) \\
& \leq(1+2 \delta)(1+\delta) C_{V}^{\prime} \Theta_{n}(\varepsilon) k^{-\varepsilon} .
\end{aligned}
$$

Then it follows from (6.2)-(6.6) that

$$
\begin{aligned}
& \left((1-2 \delta)(1-\delta) C_{V}^{\prime} \Theta_{n}(\varepsilon) / E\right)^{1 / \varepsilon}+O(1) \\
& \quad \leq N\left(E_{n}+E<H(V)<E^{\prime}\right) \\
& \quad \leq\left((1+3 \delta)(1+\delta) C_{V}^{\prime} \Theta_{n}(\varepsilon) / E\right)^{1 / \varepsilon}+O(1)
\end{aligned}
$$

as $E \searrow 0$. The arbitrariness of $\delta$ completes the proof.

\section{$\S 7 . \quad$ Proof of Theorem 1.2}

As we remarked just after Theorem 1.2, it is enough to show in the case of $z_{0}=\sqrt{-1}$.

Lemma 7.1. Let $W_{r R}$ be as in Lemma 5.2 and let $C>0$. Then we have, as $E \searrow 0$,

$$
N\left(E_{n}+E<H\left(C W_{r R}\right)<E^{\prime}\right)=\left|\frac{\log E}{2 \log R}\right|(1+o(1)) .
$$

Proof. By Lemma 3.3, we have, for any $\delta>0$ small enough,

$$
\text { (7.1) } \begin{aligned}
N\left((1-\delta) P_{n} W_{r R} P_{n}>E / C\right)+O(1) & \leq N\left(E_{n}+E<H\left(C W_{r R}\right)<E^{\prime}\right) \\
& \leq N\left((1+\delta) P_{n} W_{r R} P_{n}>E / C\right) .
\end{aligned}
$$

By Lemma 3.4 with $V=W_{r R}$, we have

$$
\text { (7.2) } \begin{aligned}
N\left((1 \pm \delta) P_{n} W_{r R} P_{n}>E / C\right) & =\sharp\left\{k \mid \gamma_{n k}\left(W_{r R}\right)>E / C(1 \pm \delta)\right\} \\
& =\sharp\left\{k \mid \log \gamma_{n k}\left(W_{r R}\right)>\log [E / C(1 \pm \delta)]\right\} .
\end{aligned}
$$


By Lemma 5.2, we have, for large $k>0$,

$$
(1+\delta) \log R^{2 k} \leq \log \gamma_{n k}\left(W_{r R}\right) \leq(1-\delta) \log R^{2 k},
$$

from which we have

$$
\begin{aligned}
& \sharp\left\{k \mid(1+\delta) \log R^{2 k}>\log [E / C(1 \pm \delta)]\right\} \\
& \quad \leq \text { the rhs of }(7.2) \\
& \quad \leq \sharp\left\{k \mid(1-\delta) \log R^{2 k}>\log [E / C(1 \pm \delta)]\right\} .
\end{aligned}
$$

On the other hand, we have

$$
\begin{aligned}
\sharp & \left\{k \mid(1 \pm \delta) \log R^{2 k}>\log [E / C(1 \mp \delta)]\right\} \\
& =\sharp\left\{k \mid k<\frac{|\log [E / C(1 \mp \delta)]|}{(1 \pm \delta)\left|\log R^{2}\right|}\right\} \\
& =\frac{1}{1 \pm \delta}\left|\frac{\log E}{\log R^{2}}\right|+O(1)
\end{aligned}
$$

as $E \searrow 0$. The result follows from (7.1)-(7.4) since $\delta>0$ is arbitrary.

We note that

$$
\begin{aligned}
\{w \in \mathbf{D}|r \leq| w \mid \leq R\} & =\{w \in \mathbf{D} \mid \log (1+r) /(1-r) \\
& \leq d(0, w) \leq \log (1+R) /(1-R)\}
\end{aligned}
$$

and $W_{r R}=F_{T, t, \sqrt{-1}}$ with $t=\log (1+r) /(1-r), T=\log (1+R) /(1-R)$ (equivalently, with $r=\tanh (t / 2), R=\tanh (T / 2))$.

Assume that $V$ satisfies $0 \leq V \leq c F_{T, 0, \sqrt{-1}}$, equivalently, $0 \leq V \leq$ $c W_{0, \tanh (T / 2)}$. Then it follows from Lemma 7.1 and the standard min-max argument for $P_{n} V P_{n}$ and $P_{n}\left(c W_{r R}\right) P_{n}$ that

$$
\begin{aligned}
\limsup _{E \searrow 0} N\left(E_{n}+E<H(V)<E^{\prime}\right) /|\log E| & \leq 1 /\left|\log R^{2}\right| \\
& =1 /\left|\log \left(\tanh ^{2}(T / 2)\right)\right| .
\end{aligned}
$$

Then the assertion (i) in Theorem 1.2 follows. The assertions (ii) and (iii) in Theorem 1.2 follow similarly in the case of $z_{0}=\sqrt{-1}$. This completes the proof.

\section{Acknowledgement}

The author would like to thank the referee for useful comments. 


\section{References}

[A-D-H] Alama, S., Deift, P. A. and Hempel, R., Eigenvalue branches of the Schrödinger operator $H-\lambda W$ in a gap of $\sigma(H)$, Comm. Math. Phys., 121, (1989), 291-321.

[A-A-R] Andrews, G.E., Askey, R. and Roy, R., Special functions, Encyclopedia Math. Appl. 71, Cambridge University Press, 1999.

[A-C-O] Antoine, M., Comtet, A. and Ouvry, S., Scattering on a hyperbolic torus in a constant magnetic field, J. Phys. A: Math. Gen., 23 (1990), 3699-3710.

[A-P] Avron, J.E. and Pnueli, A., Landau Hamiltonians on symmetric spaces, in Ideas and Methods in Quantum and Statistical Physics, vol. 2, Cambridge Univ. Press, 96-111 (1992).

[Bir] Birman, M.S., Discrete spectrum in the gaps of a continuous one for perturbations with large coupling constant, Adv. Soviet Math., 7 (1991).

[B-S] Birman, M.S. and Solomjak, M.Z., Spectral theory of self-adjoint operators in Hilbert space, D. Reidel Publishing Company, 1987.

[Com] Comtet, A., On the Landau levels on the hyperbolic space, Ann. Phys. m, 173 (1987), 185-209.

[C-H] Comtet, A. and Houston, P. J., Effective action on the hyperbolic plane in a constant external field, J. Math. Phys., 26 (1985), 185-191.

[Els] Elstrodt, J., Die Resolvente zum Eigenwertproblem der automorphen Formen in der hyperbolischen Ebene, Teil I-III, Math. Ann., 203 (1973), 295-330; Math. Z., 132 (1973), 99-134; Math. Ann., 208 (1974), 99-132.

[Fay] Fay, J., Fourier coefficients of the resolvent for a Fuchsian group, J. Reine Angew. Math., 293 (1977), 143-203.

[G-R] Gradshteyn, I. S. and Ryzhik, I. M., Tables of integrals, series, and products, Sixth edition, Jeffrey, A. (ed), Academic Press, 2000.

[Gro] Grosche, C., The path integral on the Poincaré upper half plane with a magnetic field and for the Morse potential, Ann. Phys. (N.Y.), 187 (1988), 110-134.

[Gro2] _ Path integration on the hyperbolic plane with a magnetic field, Ann. Physics, 201 (1990), 258-284.

[I-M] Ikeda N. and Matsumoto H., Brownian motion on the hyperbolic plane and Selberg trace formula, J. Funct. Anal., 163 (1999), 63-110.

[I-S] Inahama, Y. and Shirai, S., The essential spectrum of Schrödinger operators with asymptotically constant magnetic fields on the Poincaré upper-half plane, J. Math. Phys., 44 (2003), 89-106.

[K-L] Kim, S. and Lee, C., Supersymmetry-based approach to quantum particle dynamics on a curved surface with non-zero magnetic field, Ann. Physics, 296 (2002), 390-405.

[Leb] Lebedev, N. N., Special functions and their applications, Dover Publ. Inc., New York, 1990.

[M-R] Melgaard, M. and Rozenblum, G., Eigenvalue asymptotics for even-dimensional perturbed Dirac and Schrödinger operators with constant magnetic fields, Comm. Partial Differ. Equations, 28 (2003), 697-736.

[Roe] Roelcke, W., Das Eigenwertproblem der automorphen Formen in der hyperbolischen Ebene, I, Math. Ann., 167 (1966), 292-337.

[Rai] Raikov, G.D., Eigenvalue asymptotics for the Schrödinger operator with homogeneous magnetic potential and decreasing electric potential I. Behaviour near the essential spectrum tips, Comm. in P.D.E., 15 (1990), 407-434.

[Rai2] _ Border-line eigenvalue asymptotics for the Schrödinger operator with electromagnetic potential, Integral Eq. Operator Theory, 14 (1991), 875-888.

[R-W] Raikov, G.D. and Warzel, S., Quasi-classical versus non-classical spectral asymptotics for magnetic Schrödinger operators with decreasing potentials, Rev. Math. Phys., 14 (2002), 1051-1072. 
[R-S] Reed, M. and Simon, B., Methods of Modern Mathematical Physics, Vol. I-IV, Academic Press, New York, 1978.

[S-H] Saad, N. and Hall, R.L., Integrals containing confluent hypergeometric functions with applications to perturbed singular potentials, J. Phys. A., 36 (2003), 7771-7788.

[Sla] Slater, L.J., Generalized hypergeometric functions, Cambridge Univ. Press, 1966. 